\title{
Metastatic bronchial neuroendocrine tumor to the pineal gland: A unique manifestation of a rare disease
}

\author{
Simona Grozinsky-Glasberg ${ }^{1,3}$, Susana Fichman ${ }^{2}$, Ilan Shimon ${ }^{1,3}$ \\ ${ }^{1}$ Institute of Endocrinology and ${ }^{2}$ Institute of Pathology, Beilinson Hospital, Rabin Medical Center; and ${ }^{3}$ Sackler Faculty \\ of Medicine, Tel Aviv University, Israel
}

\begin{abstract}
Metastatic Neuroendocrine Tumor (NET) to the pineal gland is a unique manifestation previously unreported in the literature. We describe an unusual case of metastatic bronchial NET to the pineal gland in a 71-year-old male patient. His primary NET had been resected six years previously and there was no indication of the presence of disseminated metastatic disease at that time. Due to increased uptake by the pituitary gland on the post-operative ${ }^{111}$ Indium-pentetreotide scintigraphy (Octreoscan), an intra-sellar mass was diagnosed and excised using a transsphenoidal approach; histology revealed an unrelated non-functional pituitary macroadenoma. Four years later, a new mass appeared on MRI, involving the pineal gland, and was diagnosed on biopsy as a metastatic lesion from the original bronchial NET. Since this lesion was not accessible to surgery, it was treated successfully with radiosurgery. The case suggests that NETs should be considered in the differential diagnosis of pineal gland metastases and that radiosurgery may be an effective alternative in the treatment of these patients.
\end{abstract}

Key words: Metastatic disease, Neuroendocrine tumor, Pineal gland

\section{INTRODUCTION}

The pineal gland is located in the posterodorsal aspect of the diencephalon. Its secretory product, melatonin, has a variety of potentially important effects in humans, including anticancer, antihypertensive and antioxidant action. Moreover melatonin can act as a protector against ischemia/reperfusion injury. ${ }^{1-4}$ Primary tumors of the pineal region can be

\section{Address for correspondence:}

Simona Grozinsky-Glasberg, MD, Institute of Endocrinology, Beilinson Hospital, Rabin Medical Center, Petah Tikva, 49500, Israel, Tel: +972-3-9377182/6929,

Fax: +972-3-9211403, Email: simonag@clalit.org.il

Received 14-05-09, Revised 10-09-09, Accepted 20-11-09 of variable origin, the most commonly reported being germ cell tumors which arise from developmental abnormalities, and pineal cell tumors which develop from pinealocytes themselves. ${ }^{5}$ Metastatic tumors of the pineal gland are rarely reported in the literature, reaching a $4 \%$ incidence in patients with disseminated neoplasia originating from breast, lung or colon. ${ }^{6}$ Most metastatic pineal lesions are asymptomatic, being incidentally discovered during conventional imaging procedures for disease staging.

Bronchial Neuroendocrine Tumors (NETs) range from the well-differentiated NET to the more aggressive, poorly differentiated NET. The tumor classification is extremely important, as the latter category 
is usually associated with a worse prognosis and a shorter survival. ${ }^{7}$

To our knowledge, the present case is the first in which the pineal gland was apparently a unique metastatic site of a bronchial NET.

\section{CASE REPORT}

A 71-year-old man developed gradual pressure headaches. Six years previously he had complained of prolonged cough and hemoptysis, and a $2 \mathrm{~cm}$ lesion on the right lung was diagnosed. The patient underwent a trans-bronchial biopsy which raised the suspicion of a small cell lung carcinoma, and therefore a right pneumonectomy was performed. The pathological report revealed a NET invading the entire bronchial wall, the external aspect of the main pulmonary vessels and three hilar lymph nodes, with areas of cellular pleomorphism; the mitotic count was increased (5 mitoses/10 HPF) as was the Ki-67 proliferation index (about 20\%) without necrosis. Positive staining for neuroendocrine markers (chromogranin A, synaptophysin and neuron-specific enolase) was demonstrated on immunohistochemistry.

Following the operation, the patient was free of any NET-related symptoms, such as flushing, diarrhea, wheezing or palpitations; physical examination and laboratory tests were all normal, including complete blood count, biochemistry and 24 hours urine collection for 5-Hydroxyindoleacetic Acid (5-HIAA). An ${ }^{111}$ Indium-pentetreotide scintigraphy (Octreoscan) was then performed: this procedure usually reflects the functional accumulation of the Somatostatin (SST) analogue octreotide in tissues expressing SST receptors, such as NETs, and possesses a high diagnostic accuracy. ${ }^{8}$ The scintigraphy revealed an intense area of uptake inside the pituitary gland. A subsequent MRI of the brain discovered a $2.5 \mathrm{~cm}$ pituitary macroadenoma. Pituitary hormone tests were all normal and a transsphenoidal resection of the pituitary tumor was performed, revealing an unrelated, nonfunctional pituitary adenoma.

The patient was followed with yearly computerized tomography of the chest and brain, without any pathological finding until four years later, when a CT and a subsequent MRI of the brain revealed a $1 \mathrm{~cm}$ round lesion in the area of the pineal gland (Figure 1). The lesion did not show an uptake on repeated Octreoscan and, due to its small size, a biopsy could not be initially performed at that time. Repeated brain MRI, six months (Figure 2) and 12 months later (Figure 3), demonstrated progressive increase in the size of the pineal mass, up to $2.5 \mathrm{~cm}$. Based on tumor location, the patient was referred for an endoscopic image-guided biopsy of the pineal lesion: this is a minimally invasive and safe procedure, performed via an operating sheath and having a good diagnostic yield. In our patient this approach revealed a metastatic NET (Figure 4A), which demonstrated a lower Ki-67 proliferation index $(8 \%)$ than the primary tumor (Figure 4B). ${ }^{9}$

While pineal cells are known to naturally stain for S-antigen and synaptophysin, this latter marker together with other NET markers (such as chromogranin A and neuron-specific enolase) are prognostically important to be assessed in NETs; indeed, positive staining for chromogranin A and synaptophysin was demonstrated on immunohistochemistry in our patient's pineal metastasis (Figure 4C). ${ }^{11}$ Repeated laboratory tests for neuroendocrine markers, including 24 hours urinary collection for 5-HIAA and serum

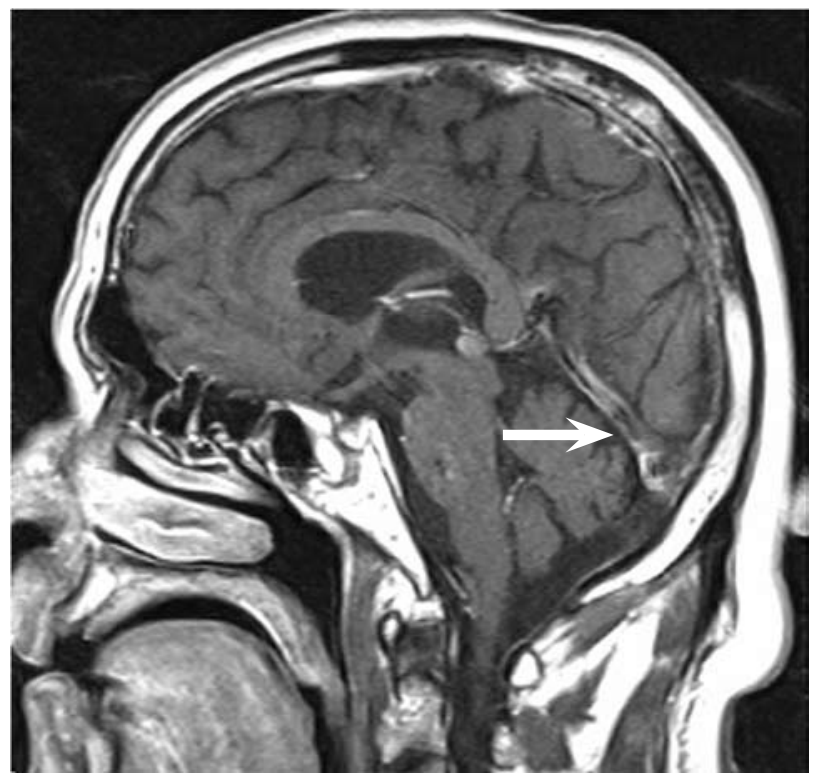

Figure 1. Gadolinium-enhanced MRI images of the brain, focused on the pineal gland (sagittal view). The arrow indicates a small lesion in the area of the base of the brain. 


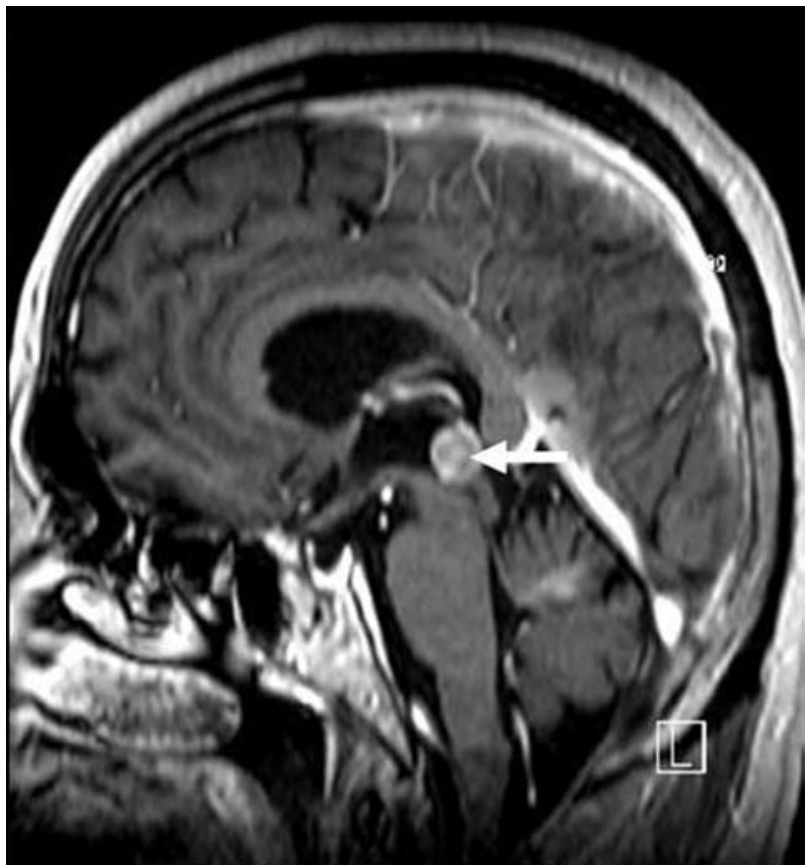

Figure 2. Gadolinium-enhanced MRI images of the brain at 6 months of diagnosis (sagittal view). Note the increase in size of the pineal mass (arrow).

Chromogranin A $(\mathrm{CgA})$, were within normal limits (5-HIAA $=3.9 \mathrm{mg} / 24 \mathrm{~h}$, normal $2-10 ; \mathrm{CgA}=47$ $\mathrm{ng} / \mathrm{ml}$, normal 19-98).

The patient was successfully treated with radiosurgery, as demonstrated by repeated MRI, performed during the follow-up period that showed tumor regression. Due to the co-existence of the bronchial

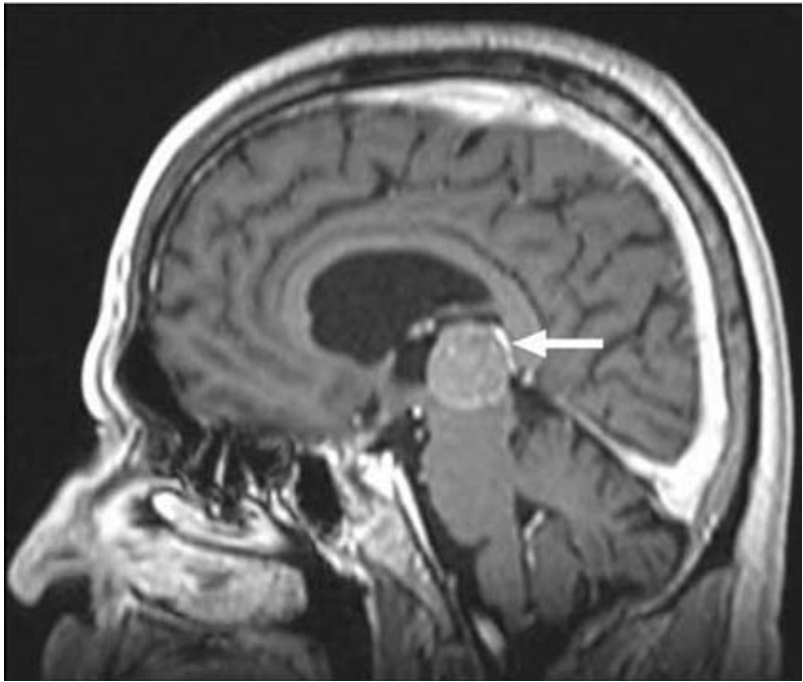

Figure 3. Repeated gadolinium-enhanced-MRI images performed just prior to the biopsy of the pineal mass (sagittal view), demonstrating that the tumor was increasing in size, starting to compress the nearby brain structures (arrow).

NET and the pituitary adenoma, genetic testing was performed which ruled out the possibility of a Multiple Endocrine Neoplasia type 1 (MEN 1) syndrome, this based on negative results for germ-line mutations of the Menin gene.

\section{DISCUSSION}

Metastatic disease to the pineal gland has seldom been described in the literature and remains a rare event. The first case of a lung carcinoma with me-
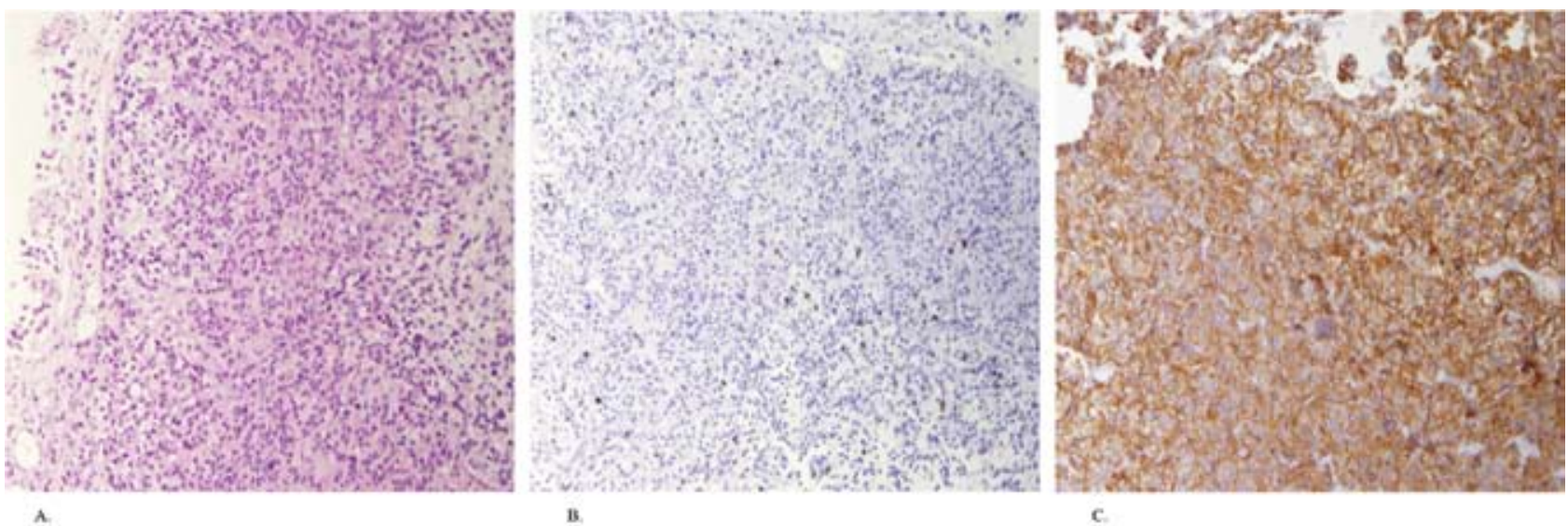

Figure 4. Histopathological characteristics of the pineal gland single metastasis. Similar to the primary lung tumor morphological and immunohistochemical characteristics, atypical NET cell appearance (A), albeit a lower KI-67 proliferation index (B), and a positive staining for synaptophysin (C) were demonstrated. 
tastasis to the pineal gland was described in 1858 by Forster, while other malignant tumors metastatic to the pineal have included breast and bronchogenic carcinomas, melanoma, renal cell, pancreatic, ovarian, gastric and frontal sinus carcinomas, in decreasing frequency. ${ }^{12,13}$ Widespread metastatic disease is the usual finding in most described cases and the time from the diagnosis of the primary tumor to the onset of Central Nervous System (CNS) symptoms (such as vertigo, confusion, headaches or vertical eye gaze paralysis) is variable. ${ }^{14-16}$ It is widely accepted that pineal involvement in these patients does not result in direct symptoms, which are usually produced through distortion of surrounding structures by pineal masses. ${ }^{17}$ Rarely, the symptoms of metastatic pineal gland involvement may precede those of the primary tumor or other metastatic sites..$^{18}$ Moreover, pineal involvement by a metastatic tumor may disturb melatonin secretion, with resultant implications for multiple processes such as immunomodulation, antioxidant capacity, stress-response, cancer protection, etc. ${ }^{19}$

Many cases of pineal metastases have been diagnosed at autopsy. The proximity of these tumors to vital brain structures limits one's ability to perform biopsies of these lesions and to reach an accurate diagnosis. ${ }^{12}$ Therefore, important factors in differentiating metastatic disease from a primary pineal gland tumor are a history of malignancy and the age of the patient, children and young adults being more frequently affected by primary pineal tumors. ${ }^{20}$

To our knowledge, the present case is the first in which the pineal gland was apparently a unique metastatic site of a neuroendocrine tumor. The patient was symptomatic, complaining of headaches. The diagnosis was based on the positive history of a primary bronchial NET, the patient's age and, mainly, on the positive findings revealed by the endoscopic biopsy of the pineal mass.

The usual approach in the management of these patients includes surgery by the Occipital Trans-Tentorial approach (OTT) followed, when necessary, by regional radiotherapy; the surgical complications are mostly transient. However, in our patient, the surgical excision of the pineal metastasis was limited by tumor size and tumor localization; therefore, the patient was referred to guided radiosurgery. ${ }^{21}$
The prognosis of patients with tumoral involvement of the pineal gland depends on the etiology and the treatment modalities. The overall mean survival time in patients with primary pineal tumors is approximately 66 months, while the reported 3 -year survival rate is about $84 \% .{ }^{21}$ However, in patients with disseminated malignancies, the survival depends on the aggressiveness of the primary tumor (based on cell morphology, mitotic rate, Ki-67 proliferation index, etc), as well as on the metastatic burden. In our patient the Ki-67 was lower in the metastatic tissue than in the primary tumour, a difference in the Ki-67 index having previously reported; this finding indicates heterogeneity in the proliferation rates between the primary and the metastatic cells, developing from a common tumoral clone. ${ }^{10}$

In conclusion, the case herein presented suggests that metastatic neuroendocrine tumors should be considered in the differential diagnosis of pineal gland tumors, particularly in elder people. It also indicates that radiosurgery may be effective in the treatment of these patients. When a metastatic tumor destroys the majority of functional pineal tissue, then consequences due to the reduction in melatonin may have to be considered. ${ }^{1-4}$ This would also be a consideration when the gland is destroyed by the treatment, as in our patient.

\section{REFERENCES}

1. Shiu SYW, 2007 Towards rational and evidence-based use of melatonin in prostate cancer prevention and treatment. J Pineal Res 43: 1-9.

2. Simko F, Paulis L, 2007 Melatonin as a potential antihypertensive treatment. J Pineal Res 42: 319-322.

3. Tengattini S, Reiter RJ, Tan DX, Terron MP, Rodella LF, Rezzani R, 2008 Cardiovascular disease: protective effects of melatonin. J Pineal Res 44: 16-25.

4. Cervantes M, Morali G, Letechipia-Vallejo G, 2008 Melatonin and ischemia- reperfusion injury of the brain. J Pineal Res 45: 1-7.

5. Lauro S, Trasatti L, Capalbo C, Mingazzini PL, Vecchione A, Bosman C, 2002 Unique pineal gland metastasis of clear cell renal carcinoma: case report and review of the literature. Anticancer Res 22: 3077-3079.

6. Kakita A, Kobayashi K, Aoki N, Eguchi I, Morita T, Takahashi H, 2003 Lung carcinoma metastasis presenting as a pineal region tumor. Neuropathology 23: 57-60.

7. Travis WD, Rush W, Flieder DB, et al, 1998 Survival analysis of 200 pulmonary neuroendocrine tumors with 
clarification of criteria for atypical carcinoid and its separation from typical carcinoid. Am J Surg Pathol 22: 934-944.

8. Granberg D, Sundin A, Janson ET, Oberg K, Skogseid B, Westlin JE, 2003 Octreoscan in patients with bronchial carcinoid tumours. Clin Endocrinol (Oxf) 59: 793-799.

9. Pople IK, Athanasiou TC, Sandeman DR, Coakham HB, 2001 The role of endoscopic biopsy and third ventriculostomy in the management of pineal region tumours. Br J Neurosurg 15: 305-311.

10. Matheus RS, Bernardi F del C, Gallo CP, et al, 2004 Nuclear markers (star volume, mitotic index, AgNOR and $\mathrm{Ki}-67$ ) of the primary tumor and its metastasis in non-small cell lung carcinomas. Pathol Res Pract 200: 13-23.

11. Redecker P, Bargsten G, 1993 Synaptophysin - a common constituent of presumptive secretory microvesicles in the mammalian pinealocyte: a study of rat and gerbil pineal glands. J Neurosci Res 34: 79-96.

12. Weber P, Shepard KV, Vijayakumar S, 1989 Metastases to pineal gland. Cancer 63: 164-165.

13. Holness RO, Sangalang VE, 1976 Myelomatous metas- tasis to the pineal body. Surg Neurol 5: 97-100.

14. Ouyang R, Rozdilsky B, 1966 Metastasis of carcinoma to pineal body. Report of two cases. Arch Neurol 15: 399-403.

15. Lassman AB, Bruce JN, Fetell MR, 2006 Metastases to the pineal gland. Neurology 67: 1303-1304.

16. So SC, 1976 Pineal tumours: a clinical study of 23 cases. Aust N Z J Surg 46: 75-79.

17. Ortega P, Malamud N, Shimkin MB, 1951 Metastasis to the pineal body. AMA Arch Pathol 52: 518-528.

18. Ahn JY, Chung YS, Kwon SO, Huh R, Chung SS, 2005 Isolated pineal region metastasis of small cell lung cancer. J Clin Neurosci 12: 691-693.

19. Srinivasan V, Maestroni GJ, Cardinali DP, Esquifino AI, Pandi Perumal SR, Miller SC, 2005 Melatonin, immune function and aging. Immun Ageing 2: 17.

20. Tomita T, Wetzel N, 1984 Metastasis to the midbrain. Report of two cases. J Neurooncol 2: 73-77.

21. Cho BK, Wang KC, Nam DH, Kim DG, Jung HW, Kim HJ, 1998 Pineal tumors: experience with 48 cases over 10 years. Childs Nerv Syst 14: 53-58. 